\title{
Intermittent visual information and the multiple time scales of visual motor control of continuous isometric force production
}

\author{
JACOB J. SOSNOFF and KARL M. NEWELL \\ Pennsylvania State University, University Park, Pennsylvania
}

\begin{abstract}
In an experiment, we examined the effect of intermittency (from $25.6 \mathrm{~Hz}$ to $0.2 \mathrm{~Hz}$ ) of visual information on continuous isometric force production as a function of force level $(5 \%, 10 \%, 25 \%$, and $50 \%$ of maximal voluntary contraction [MVC]). The amount of force variability decreased and the irregularity of force output increased as a function of increased visual intermittency rate. Vision was found to have an influence on the frequency structure of force output up to $12 \mathrm{~Hz}$, and the $25 \%$ MVC force level had more high-frequency modulations with higher rates of visual information. The effective use of intermittent visual information is mediated nonlinearly by force level, and there are multiple time scales of visual control (range, $\sim 0-12 \mathrm{~Hz}$ ) that are postulated to be a function of both feedback and feedforward control processes.
\end{abstract}

A longstanding issue in motor control is whether the processing of visual motor information is continuous or intermittent (Carlton, 1992; Elliott, 1990; Thomson, 1980, 1983). This debate has its origins in traditional and contemporary models of human information processing and their assumptions about discrete and continuous flow of information (Broadbent, 1958; Miller, 1988; Sanders, 1990). The significance of the discrete versus continuous processing debate has been neutralized of late by the realization that the question can lead to false dichotomies and that the answer is dependent on task specificity (Mulder \& van Galen, 1995). Nevertheless, a continuing and related challenge is to determine how intermittent visual information is processed in the apparent continuous control of movement or force output (Elliott, 1990, 1992; Elliott, Carson, Goodman, \& Chua, 1991; von Hofsten \& Lee, 1982).

The predominant experimental approach to the intermittent or continuous visual control of movement has been to determine the minimal visual-processing time for limb control (see the review by Carlton, 1992). The experiments tend to follow variations of a protocol initiated by Woodworth (1899) with which the minimal movement time that is uninfluenced by the absence or withdrawal of vision is investigated (e.g., Carlton, 1981; Keele \& Posner, 1968; Vince, 1948). The estimates of the minimal visualprocessing time have decreased from Woodworth's initial

This research was supported in part by funding from the National Institutes of Health (T32-AG-00048). The authors thank D. Elliott and an anonymous reviewer for their helpful comments in revising the article. We also thank Tim Benner for his technical assistance. Correspondence concerning this article should be addressed to J. J. Sosnoff, Department of Kinesiology, Pennsylvania State University, 266 Recreation Hall, University Park, PA 16802 (e-mail: jjs388@psu.edu). estimate of $400 \mathrm{msec}$, so that the contemporary position is that it is much less than a visual reaction time and on the order of about $100 \mathrm{msec}$ (Smith \& Bowen, 1980; Zelaznik, Hawkins, \& Kisselburgh, 1983).

Clearly, the estimate of minimal visual-processing time is influenced by the nature of the movement task and the experimental strategy used to measure the influence of the visual input (e.g., accuracy measures, determination of an amendment to the ongoing movement trajectory, and so on). The discrete visual-processing paradigm tends to emphasize the determination of the minimal feedback loop time that influences the control of movement kinematics, but this approach does not rule out the possible feedforward anticipatory uses of vision (Khan et al., 2003). Indeed, in many tasks, both feedback and feedforward processes are in effect, and so visual motor control involves multiple processes and time scales beyond that of just reflecting the minimal movement time that is uninfluenced by vision (Desmurget \& Grafton, 2000; Newell, Slobounov, Slobounova, \& Molenaar, 1997).

Another approach to the assessment of the visual control of movement is to manipulate the intermittency of the presentation of visual information in the maintenance of a steady state position or force level (Slifkin, Vaillancourt, \& Newell, 2000). Here, the stability properties of the action are highly reliable, and the change in the force output or movement is minimal, both of which are features that should enhance the speed of visual motor information processing. In addition, modern computer protocols allow the manipulation of the intermittency of vision in such tasks over finely controlled temporal intervals while, at the same time, affording precise measures of the continuous motor output.

Slifkin et al. (2000) manipulated the intermittency (frequency) of visual information presentation in an iso- 
metric force task, where the participant was attempting to maintain a constant level of force output. The presence of visual information was manipulated over a range from 0.2 to $25.6 \mathrm{~Hz}$, and performance outcome improved as the rate of presentation of vision increased from the most intermittent $(0.2 \mathrm{~Hz})$ up to a $6.4-\mathrm{Hz}$ feedback frequency (about $160 \mathrm{msec}$ ). Spectral analysis showed that vision had no impact on the frequency structure of force output higher than about the 1-Hz range (see also Freund \& Hefter, 1993; Miall, Weir, \& Stein, 1993), a finding that is consistent with the long-held idea that there is a memory buffer for the accumulation of visual information before the amendment of movement (Adams \& Dijkstra, 1966). These results led Slifkin and colleagues to propose a model of isometric force control in which error information is sampled at a rate of $6.4 \mathrm{~Hz}$ (every $160 \mathrm{msec}$ ) and stored and, then, a motor correction is made once per second $(1 \mathrm{~Hz})$ on the basis of the stored information.

There are a number of theoretical concerns with the relation proposed by Slifkin et al. (2000) between the intermittency of visual input and that of the motor output. First, the model was based on the findings of force output at a single force level (40\% maximal voluntary contraction $[\mathrm{MVC}]$ ), and it has been shown that the level of force output mediates nonlinearly the visually induced modulations of force output (Slifkin \& Newell, 1999, 2000). Furthermore, the inference that movement (force) corrections can only be made at $1 \mathrm{~Hz}$ seems contrary to the fact that modulations in movements and force output can be made at much faster rates. Since there is generally an inverse relationship between force amplitude and frequency, it is possible that the modal $1-\mathrm{Hz}$ component of the model is a result of the moderate force level used in the investigation, in that faster frequency force modulations are more persistent and apparent at lower force levels.

The $1-\mathrm{Hz}$ output of the model is further complicated by recent findings from our laboratory, which show that there is not a modal frequency of force output (Deutsch \& Newell, 2001; Mayer-Kress, Deutsch, \& Newell, 2003; Mayer-Kress \& Newell, 2002). Rather, the force output appears to be a fractal $1 / f$ noise-like process containing both deterministic and stochastic components at multiple time scales (i.e., low and high frequencies). This finding is consistent with a growing variety of other data sets revealing $1 / f$-like cognitive motor processes (Gilden, Thorton, \& Mallon, 1995; Ward, 2002).

The purpose of this investigation was to examine (1) the generalization of Slifkin et al.'s (2000) vision intermittency model across a range of submaximal force levels and (2) the effect of visual intermittency on the multiple time scales of continuous force output. In order to address these empirical questions, participants were asked to produce isometric force (force from essentially no positional change of the limb) at four distinct constant force levels $(5 \%, 10 \%, 25 \%$, and $50 \%$ MVC) with varying levels of visual intermittency ranging from $25.6 \mathrm{~Hz}$ (40 $\mathrm{msec})$ to $0.2 \mathrm{~Hz}(5,000 \mathrm{msec})$. The general hypothesis tested was that the frequency structure of the intermittent visual information will interact with force level in determining both the amount and the structure of force variability over multiple time scales. Specifically, it was predicted that the amount of visual information needed to maximize performance would be dependent on force level, with lower magnitude force output being modulated by higher rates of visual intermittency in a $1 / f$-like process.

\section{METHOD}

\section{Participants}

Eleven participants (4 males and 7 females) from the Pennsylvania State University community, with a mean age of $24.6 \pm$ 2.8 years, volunteered to take part in the experiment. All of the participants were right-hand dominant, free of neuromuscular disease, and had normal or corrected-to-normal vision. The participants performed the task with their right index fingers. The participants signed informed consent forms approved by the local Institutional Review Board.

\section{Apparatus}

Each participant was seated approximately $60 \mathrm{~cm}$ in front of a 17-in computer monitor (Digiview), with his or her dominant hand placed on the table. An Eltran EL-500 load cell (diameter, $1.27 \mathrm{~cm}$ ) that measured compressed forces with an accuracy of $0.0015 \mathrm{~N}$ was fixed $45 \mathrm{~cm}$ in front of the participant's midline. The voltage output of the load cell was amplified by a Coulbourn (V72-25) resistive bridge strain amplifier with an excitation voltage of $10 \mathrm{~V}$ and an amplifier gain of 100 and then was sampled at $100 \mathrm{~Hz}$ by a $16-$ bit A/D board. The computer monitor had a viewing area of $800 \times$ 600 pixels and a dot pitch width (the width of each pixel) of $\sim 0.07 \mathrm{~mm}$. The display to control gain was set at 50 pixels $/ \mathrm{N}$.

During each trial, the participant produced force isometrically with the distal phalanx of the dominant index finger to match a force target that was displayed as a red horizontal line (1 pixel wide) spanning the width of the screen. Although no physical restraint was used, the participant was instructed to keep his or her nonactive fingers, forearm, and wrist flat on the table throughout the experiment.

\section{Procedure}

Estimation of maximal voluntary contraction. The participant's MVC strength was determined at the start of the experimental session. The participant was instructed to produce the maximal amount of isometric force possible by flexing the index finger. The participant produced maximal force while pushing against the load cell. The force applied to the load cell was displayed on the monitor to provide visual feedback. Three 6-sec maximal contractions were recorded, with a $30-\mathrm{sec}$ rest between each contraction. The participant's MVC was determined to be the average of the highest force produced in each trial.

Experimental design and instructions. Overall, the procedures and equipment utilized were similar to those in Slifkin et al. (2000). In brief, the participant adjusted his or her force output to match a red target line displayed on the monitor and viewed on-line feedback of his or her performance in the form of a series of yellow dots, which corresponded to the force trajectory that moved from left to right across the screen with time. Once presented, each yellow dot stayed illuminated on the screen until $6 \mathrm{sec}$ after the completion of the trial. The target line corresponded to $5 \%, 10 \%, 25 \%$, or $50 \%$ of the participant's MVC. The frequency at which the force trajectory was displayed was experimentally manipulated. Eight distinct frequencies of intermittent visual feedback $(0.2,0.4,0.8,1.6,3.2,6.4$, 12.8 , and $25.6 \mathrm{~Hz}$ ) were presented at each force level. For example, in the $25.6-\mathrm{Hz}$ condition, visual feedback corresponding to the force output was presented every $40 \mathrm{msec}$, whereas in the $0.2 \mathrm{~Hz}$ condition, visual information was presented every 5,000 msec. 
Force Output

A

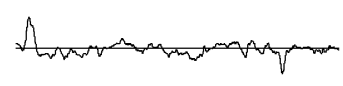

B

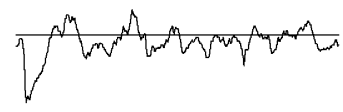

C
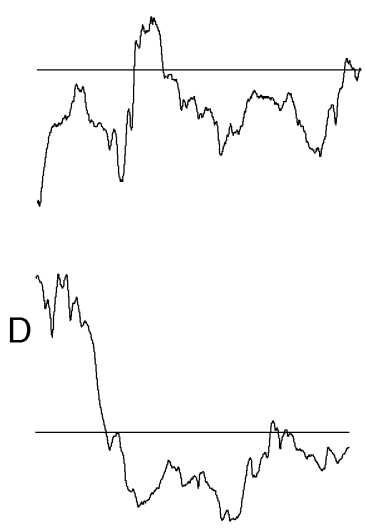

Visual Feedback

$25.6 \mathrm{~Hz}$

$1 \mathrm{~N}$

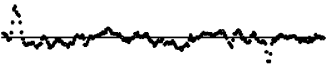

$2 \mathrm{sec}$

$6.4 \mathrm{~Hz}$

$1 \mathrm{~N}$
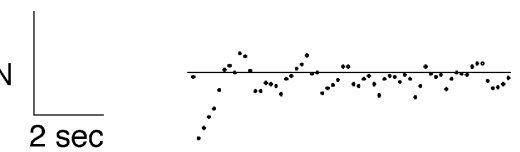

$0.8 \mathrm{~Hz}$

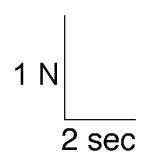

$0.2 \mathrm{~Hz}$

$1 \mathrm{~N}$

$2 \mathrm{sec}$
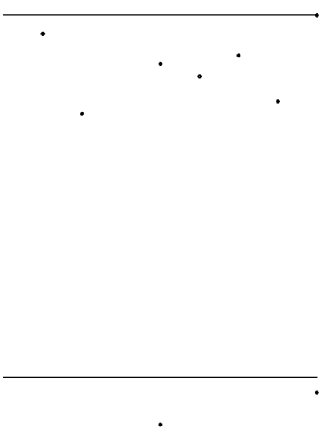

Figure 1. The force output of a representative participant at $10 \%$ maximal voluntary contraction at four different rates of visual feedback (from top to bottom: 25.6, 6.4, 0.8 , and $0.2 \mathrm{~Hz}$, respectively) is shown in the right column. The straight line is the force target; the jagged line is the actual force output. The intermittent visual feedback presented to the participant for each unique feedback condition is shown in the left column.

The left-hand column of Figures 1A-1D shows a representative participant's force output at a $10 \%$ force level at four different intermittencies (25.6, 6.4, 0.8, and $0.2 \mathrm{~Hz}$, respectively). The right-hand column of the figure depicts the visual display presented to the participant at each respective intermittency condition. Three 15 -sec trials were performed at each of the 32 unique force-frequency conditions.

Experimental sessions were held on two separate days in a 7-day period. Each session consisted of two blocks of 24 trials of one high ( $25 \%$ or $50 \%$ MVC) and one low ( $5 \%$ or $10 \%$ MVC) force level. The eight frequency conditions were randomly presented within the block of trials at each force level. In order to minimize fatigue, the participant was given a 20 -sec rest between trials.

The participant was instructed to minimize the deviations between the yellow force trajectory and the target line throughout all the trials. The participant was presented a feedback score at the end of the trial to encourage performance. The score was the root-mean square (RMS) error and was calculated with $\left[\Sigma\left(s_{i}-f_{i}\right)^{2 / n}-1\right]^{1 / 2}$, where $s_{i}$ is the $i$ th value of the target, $f_{i}$ is the $i$ th force sample, and $n$ is the number of data samples. It was based on the performance during the last $10 \mathrm{sec}$ of the trial.

\section{Data Analysis}

The initial $4 \mathrm{sec}$ and the final $1 \mathrm{sec}$ of force data from each trial were removed prior to analysis to avoid the initial force stabilization and/or premature cessation of force production. The $10 \mathrm{sec}$ of force data were lowpass filtered with a cutoff of $30 \mathrm{~Hz}$ (9th order Butterworth). All data processing was performed using software written in Matlab 6.1 (Mathworks, 1999).

\section{Measures of Task Performance}

In order to assess task performance as a function of force and visual feedback, the mean, the standard deviation, and a measure of information transfer of the force data were calculated. The signal-tonoise ratio (mean/SD) was used as a measure of information transfer (Slifkin et al., 2000).

\section{Structure of Force Output}

In order to examine how varying levels of visual feedback influenced the structure of force output across separate force levels, both time and frequency domain analyses were performed. The time domain structure of force output was assessed by approximate entropy 
(ApEn; Pincus, 1991) and detrended fluctuation analysis (DFA) (Peng et al., 1993). Spectral analysis was performed on the force data in order to examine how the frequency structure of the force signal changed with the intermittency of the visual feedback levels. Since the calculation of ApEn and spectral analysis can be biased by the presence of nonstationarities, the data were linearly de-

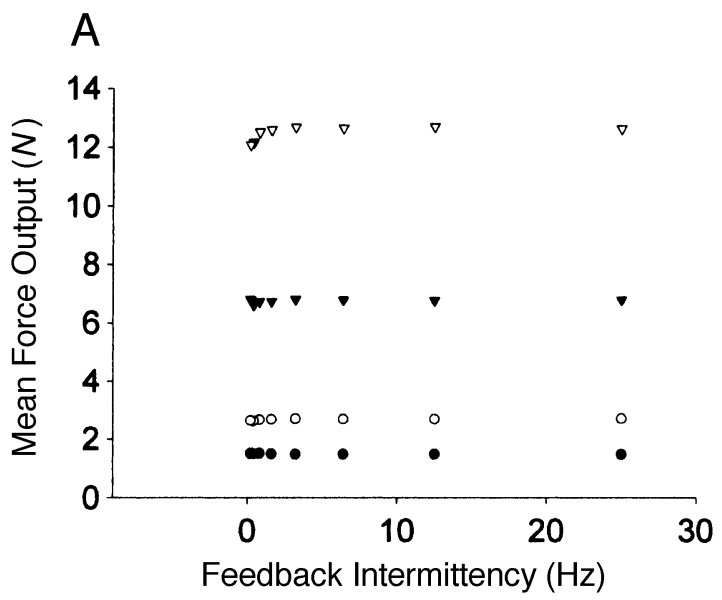

B
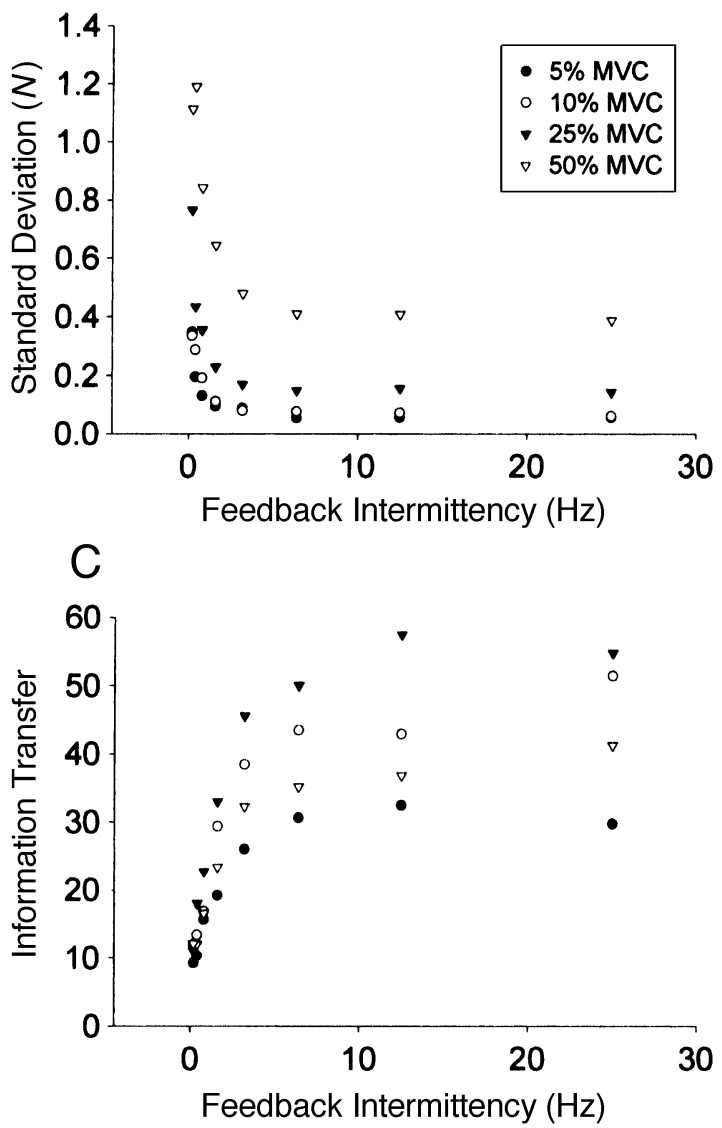

Figure 2. (A) Mean force output as a function of feedback intermittency for each force level. (B) Standard deviation of force output as a function of feedback intermittency for each force level. (C) Information transfer of force output as a function of feedback intermittency for each force level. MVC, maximal voluntary contraction. trended (i.e., the mean of the data was subtracted from the time series) prior to these analyses.

ApEn yields a single value that quantifies the regularity (complexity) of a time series. A very regular signal, such as an ideal sine wave, would have an ApEn value approaching 0, whereas a random time series (white noise) would have a value close to 2 . Thus, increases in ApEn are said to reflect increase in the signal's time domain complexity (Pincus, 1991). The appendix in Slifkin and Newell (1999) provides a detailed explanation of how ApEn is calculated.

DFA detects long-range correlations in nonstationary data (Peng et al., 1993). The full methods of how to calculate DFA have been previously reported (Hausdorff et al., 1997). Briefly, the force signal is first integrated and then detrended. Next, the RMS of the modified force signal is determined at different time scales. The slope of the relationship between the RMS (fluctuation magnitude) and the time scale is deemed the fractal scaling index $(\alpha)$. Importantly, $\alpha$ depends on the sequential ordering of the fluctuations within the data, and not on their magnitude. If the force fluctuations are random (i.e., completely independent), $\alpha$ would tend to a value of 0.5 . A value of 1.5 would suggest that the force signal was similar to Brown noise. If the force fluctuations are correlated across multiple time scales, the value of $\alpha$ would approximate 1. Previous work has shown that constant isometric force produced with full visual feedback has an $\alpha$ of approximately 1.25 (Vaillancourt, Larsson, \& Newell, 2003).

The power spectrum was computed using the PSD command in Matlab 6.1 that uses Welch's averaged periodogram method. A 256point nonoverlapping Hanning window was used, with a sampling frequency of $100 \mathrm{~Hz}$, resulting in a $0.39 \mathrm{~Hz}$ binwidth. The power in each bin represented the amplitude of force oscillations that occur at the frequency specified by the bin. Changes in power as a function of force level and visual information were calculated in two distinct ways. First, the amount of power in $2-\mathrm{Hz}$ bandwidths from $0-2$ to $10-12 \mathrm{~Hz}$ was calculated. This frequency range accounted for approximately $99 \%$ of the power within the spectrum. Second, a power function of the form $P=a\left(f^{\beta}\right)$ was fitted to the spectral profile. In this equation, $\beta$, the power function exponent scales changes in spectral frequency $(f)$ to changes in spectral power $(P)$. When plotted, $\beta$ identifies the slope or rate of change of spectral power as a function of spectral frequency within a spectral profile, while $a$ represents the $y$ intercept (Lipsitz, 1995; Slifkin et al., $2000)$. For the purposes of the present experiment, only $\beta$ is of interest, and as the power spectrum becomes more broadband, the value of $\beta$ will increase from negative values toward zero. Consequently, spectral slope analysis provides a method by which to examine the global structure of force output.

\section{Statistical Analysis}

Each of the dependent variables discussed above was placed independently in a two-way $(4 \times 8)$ repeated measures analysis of variance (ANOVA) with force level and visual intermittency as the factors. When relevant, Tukey's honestly significant difference (HSD) test was used to determine the specific effects contributing to the general ANOVA. All statistics were evaluated as significant when there was less than a $5 \%$ chance of making a Type I error $(p<$ $.05)$. When a nonsignificant trend was observed, the effect size as indexed by $\eta^{2}$ was reported, which quantifies the proportion of variance that is accounted for by a change in a factor. Traditionally, $\eta^{2}$ equal to or greater than .14 is interpreted as a large effect size (Green \& Salkind, 2003). All statistical analyses were completed using Statistica statistical package (StatSoft Inc., Tulsa, OK).

\section{RESULTS}

\section{Task Performance: Isometric Force Production}

Maximal force output (MVC) ranged from 17.63 to $37.85 \mathrm{~N}(27.13 \pm 6.36 \mathrm{~N})$. Figure 2 A illustrates the finding 
that participants scaled their force output to the four target levels [5\% MVC $(1.49 \mathrm{~N})$ vs. $10 \% \operatorname{MVC}(2.67 \mathrm{~N})$ vs. $25 \%$ $\operatorname{MVC}(6.75 \mathrm{~N})$ vs. $50 \% \operatorname{MVC}(12.5 \mathrm{~N}) ; F(3,30)=140.4$, $p<.05]$. Further investigation of mean force output revealed a two-way interaction between force level and visual intermittency $[F(21,210)=3.93, p<.05]$. The interaction was found to be a result of an approximate $0.2-0.5 \mathrm{~N}$ decrease in mean force output as a function of decreased intermittency rates, but only at the higher force levels $(25 \%$ and $50 \% \mathrm{MVC}$ ).

The effects of visual intermittency on the standard deviation $(S D)$ of force output are shown in Figure 2B. It is clear from the figure that $S D$ is greater in the higher force levels $[F(3,30)=87.73, p<.05]$ and decreases as a function of greater rates of intermittency $[F(7,70)=$ $51.08, p<.05]$. Statistical analysis also revealed an interaction between force level and visual intermittency $[F(21,210)=9.95, p<.05]$. Post hoc analysis showed that the level of intermittency at which $S D$ was statistically different from higher intermittency rates was dependent on force level. For instance, in the lowest force level, $S D$
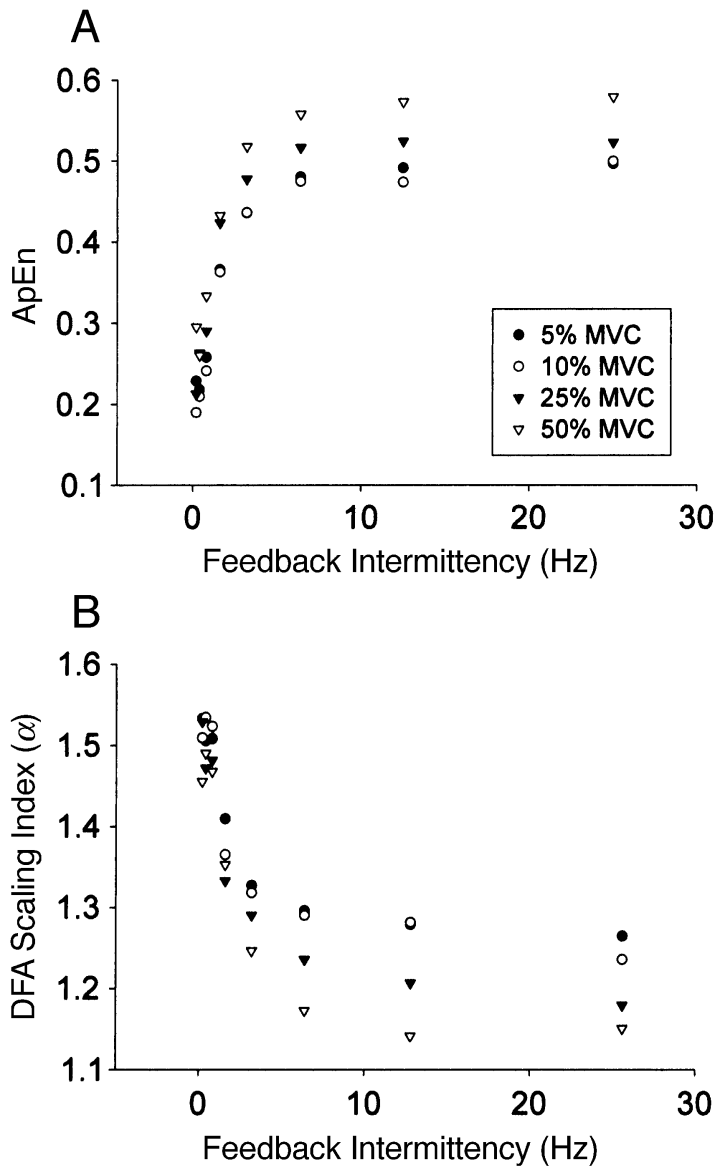

Figure 3. (A) Approximate entropy (ApEn) of force output as a function of feedback intermittency for each force level. (B) Detrended fluctuation analysis (DFA) fractal scaling index $(\alpha)$ of force output as a function of feedback intermittency for each force level. MVC, maximal voluntary contraction. was greater in the $0.2-\mathrm{Hz}$ intermittency condition than in all the other intermittency conditions, whereas in the highest force task, all the conditions with intermittency rates less than $3.2 \mathrm{~Hz}$ had larger $S D$ s (see Figure 2A).

Figure $2 \mathrm{C}$ shows that information transfer increases as a function of visual intermittency $[F(7,70)=63.14$, $p<.05]$. It is noticeable in the figure that the $25 \% \mathrm{MVC}$ task had the greatest information transfer $[F(3,30)=$ $15.27, p<.05]$. A force level $\times$ intermittency interaction was also revealed by statistical analysis $[F(21,210)=$ $4.46, p<.05]$. This interaction was found to be due to the lack of a difference in information transfer between force levels at the lower intermittency rates $(0.2,0.4$, and $0.8 \mathrm{~Hz}$ ) and the systematic force level effect at higher rates of intermittency.

In summary, analysis of task performance revealed three important findings. First, mean force output decreased a small but significant amount as a function of decreased intermittency of visual information, but only at the higher force levels. Second, the visual intermittency rate at which $S D$ increased was dependent on force level. Third, there was no difference between force levels in information transfer at the smallest rates of intermittency. Overall, these results support the hypothesis proposed in the introduction that visual motor processing is dependent on the magnitude of force output. The amount of visual information needed to maximize performance is dependent on force level, with lower magnitude force output being modulated by greater visual feedback frequency.

\section{Structure of Force Output}

Figure $3 \mathrm{~A}$ shows that the irregularity (ApEn) of the force output increases as a function of visual feedback frequency across all force levels $[F(7,70)=107.32, p<.05]$. It is also noticeable that the higher force levels are more irregular and that there are minimal differences between the two lower force levels $[F(7,70)=5.79, p<.05]$.

The findings of the ApEn analysis are congruent with the DFA calculations, which are depicted in Figure 3B. Specifically, the DFA scaling exponent decreased from $\sim 1.5$ to $\sim 1.1$ as the intermittency rate increased $[F(7,70)=$ $86.9, p<.05]$. This suggests that isometric force production with minimal vision approximates Brownian motion but has more significant long-range correlations at multiple time scales when vision is available. In addition, it was found that the lower force levels had a greater scaling exponent (1.39 and 1.38) than the higher force levels did $[1.34$ and $1.31 ; F(3,30)=6.60, p<.05]$.

To further examine the structure of the force output as a function of visual information, a frequency analysis was performed on the force output. Figure 4 shows two representative power spectra of a single participant at $10 \% \mathrm{MVC}$ at a high $(25.6 \mathrm{~Hz})$ and a low $(0.2 \mathrm{~Hz})$ rate of visual feedback. The spectrum from the high rate of visual feedback is depicted as a solid line, while the low rate of feedback spectrum is depicted with a dashed line. The most obvious difference between the two spectra is the large reduction in magnitude of power around $1 \mathrm{~Hz}$ 


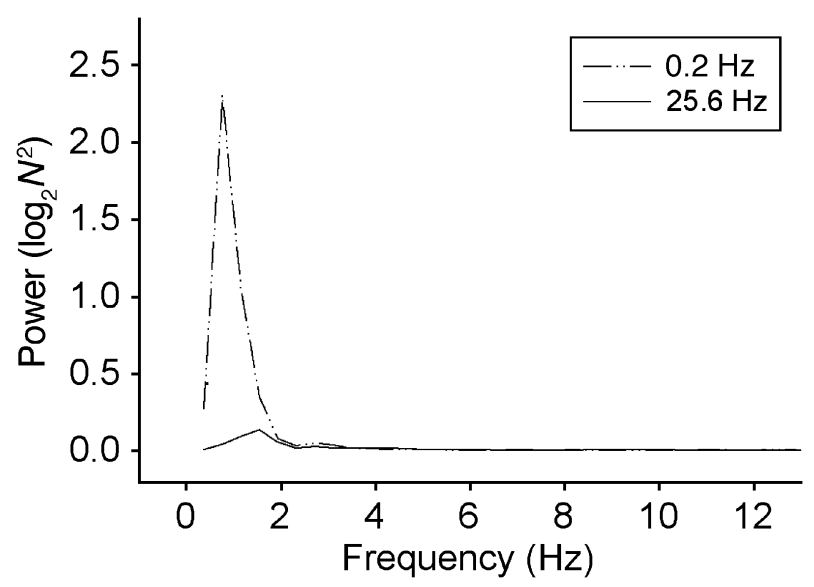

Figure 4. Frequency profile of a representative participant's (same as in Figure 1) force output at $10 \%$ maximal voluntary contraction with a visual feedback rate of $25.6 \mathrm{~Hz}$ (solid line) and $0.2 \mathrm{~Hz}$ (dotted line).

with the decrease in rate of visual intermittency. In order to quantify these changes in the force spectrum as a function of visual intermittency, spectral slope, as well as the average amount of power in $2-\mathrm{Hz}$ bandwidths from 0 to $12 \mathrm{~Hz}$, was calculated.

The spectral slope gives a global index of the broadness of the force spectrum, with a value closer to 0 being relatively broader. Figure 5 shows that the spectral slope becomes less negative with an increase in visual intermittency rate ( - 2.8 vs. $\sim-2.4)$ across all force levels $[F(7,70)=26.80, p<.05]$. Although it appears in the figure that there is a main effect of force, with the $50 \%$ force level having the largest (least negative) spectral slope, it did not reach the traditional levels of significance $\left(p=.11, \eta^{2}=.18\right)$.

The spectral slope analysis revealed that the force spectrum became more broadband as visual feedback intermittency was reduced. This analysis is not informa-

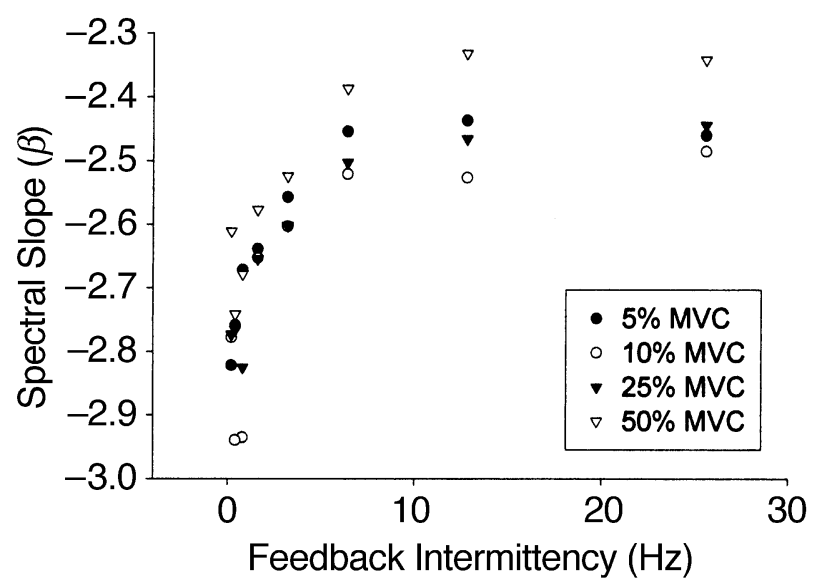

Figure 5. Spectral slope $(\beta)$ of force output as a function of feedback intermittency for each force level. MVC, maximal voluntary contraction. tive about the frequency band within the spectrum in which this broadening takes place. In order to find where in the power spectrum vision influences the amount of power, the average amount of power in $2-\mathrm{Hz}$ bandwidths was determined. Figures $6 \mathrm{~A}-6 \mathrm{~F}$ depict the average power from 0 to $12 \mathrm{~Hz}$ in $2-\mathrm{Hz}$ bandwidths as a function of visual feedback frequency at all four force levels. For aesthetic purposes, the power and intermittency rate were transformed with a $\log _{10}$ transform in the figure. As was expected, the higher force levels had greater power in all bandwidths $(p<.05)$. Visual inspection of Figure 6 also suggests that there was an influence of visual intermittency out to $12 \mathrm{~Hz}$. Statistical analysis revealed that there was a decrease in power with increased visual intermittency rate in the 0 - to 2-, 2- to 4-, 4- to 6-, and $6-$ to $8-\mathrm{Hz}$ bandwidths $(p<.05)$. Although not significant, there was also a trend for an effect of vision in the 8 to $10-\mathrm{Hz}$ bandwidth ( $p=.09, \eta^{2}=.16$ ).

To further validate the effect of visual feedback on specific regions of the force spectrum, linear regression was performed. In Table 1, the intercept, slope, and percentage of variance accounted for $\left(r^{2}\right)$ for each regression equation are listed. Overall, it was found that decreasing intermittency of vision led to a decrease in power (a negative slope) in all of the bandwidths up to $12 \mathrm{~Hz}$. More specifically, decreasing intermittency was found to decrease power up to $4 \mathrm{~Hz}$ for all the force levels. Intermittency was found to have an effect only in the 6 - and $8-\mathrm{Hz}$ bandwidths for the $5 \%, 10 \%$, and $25 \% \mathrm{MVC}$ conditions. Power decreased in the $10-\mathrm{Hz}$ bandwidth with decreasing intermittency only in the $10 \%$ and $25 \%$ MVC conditions. Lastly, only in the $25 \%$ MVC condition was power decreased in the $12-\mathrm{Hz}$ bandwidth as a function of visual intermittency. It was also found that vision accounted for a greater amount of variance in the force spectrum at the $25 \%$ force levels (see Table 1). In addition, Table 1 shows that the change in power as a function of intermittency (i.e., the slope) was smallest at the highest force level.

In order to further isolate the frequencies in the force spectrum that are influenced by vision, the amount of power in each $0.39-\mathrm{Hz}$ bin from the frequency analysis was calculated. The amount of power in all of the frequency bins from 0.39 to $3.90 \mathrm{~Hz}$ was found to be influenced by vision, so that increases in visual feedback rate led to decreases in power in these bandwidths. In addition, power in the 5.85-, 6.63-, 7.09-, 7.41-, and 12.10$\mathrm{Hz}$ bands was found to statistically decrease with increases in the visual intermittency rate $(p<.05)$.

The examination of the frequency structure of the force signal as a function of force level and visual intermittency revealed several important findings. It was observed that there was greater magnitude of power at the higher force levels. Coarse analysis with a bin resolution of $2 \mathrm{~Hz}$ showed that power decreased as the visual feedback rate increased up to $8 \mathrm{~Hz}$, with the decrease in power being most apparent at the $25 \%$ MVC condition. However, finer resolution frequency analysis revealed that power in bandwidths from 0 to $3.90,6$ to 7.5 , and 11.71 to $12.10 \mathrm{~Hz}$ de- 
- $5 \%$ MVC

- $10 \%$ MVC

- $25 \%$ MVC

$50 \%$ MVC
A $0-$ to $2-\mathrm{Hz}$ Bandwidth

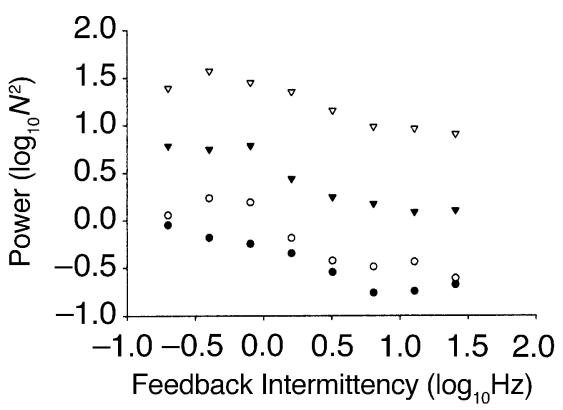

C 4- to 6- $\mathrm{Hz}$ Bandwidth

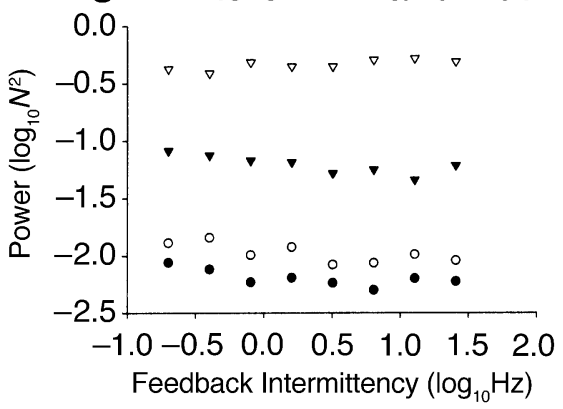

E 8- to $10-\mathrm{Hz}$ Bandwidth

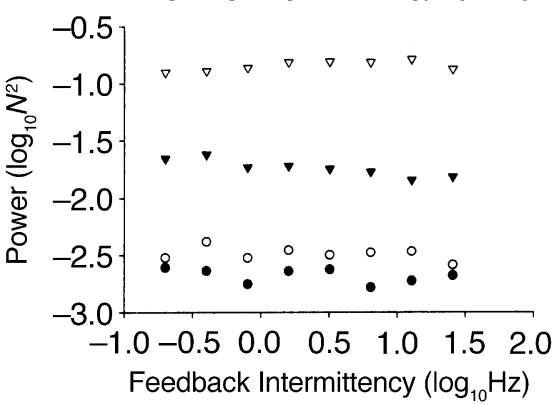

B 2- to 4- $\mathrm{Hz}$ Bandwidth

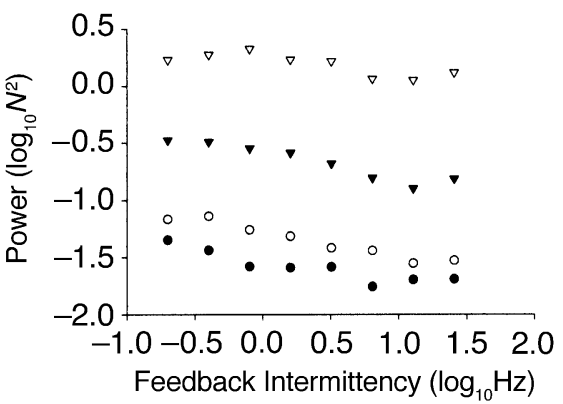

D 6- to 8- $\mathrm{Hz}$ Bandwidth

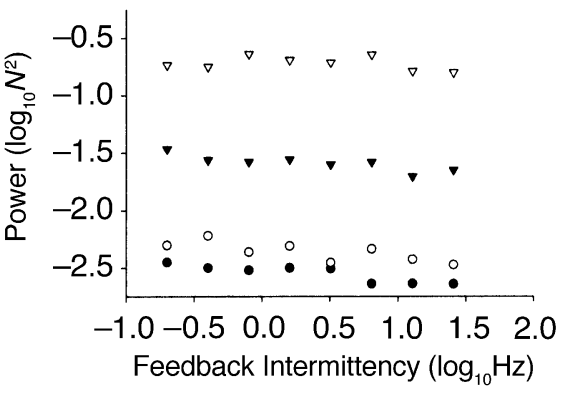

F 10- to $12-\mathrm{Hz}$ Bandwidth

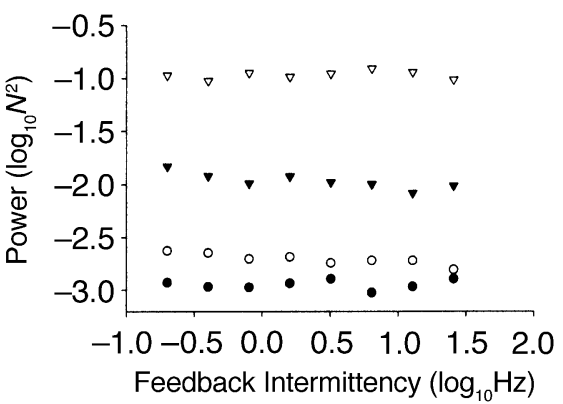

Figure 6. (A) Average amount of power in the force spectrum as a function of feedback intermittency at $10 \%$ maximal voluntary contraction (MVC) in the 0 to $2-\mathrm{Hz}$ bandwidth. (B) Average amount of power in the force spectrum as a function of feedback intermittency at $10 \% \mathrm{MVC}$ in the 2 to $4-\mathrm{Hz}$ bandwidth. (C) Average amount of power in the force spectrum as a function of feedback intermittency at $10 \%$ MVC in the 4 to 6-Hz bandwidth. (D) Average amount of power in the force spectrum as a function of feedback intermittency at $10 \%$ MVC in the 6 to 8-Hz bandwidth. (E) Average amount of power in the force spectrum as a function of feedback intermittency at $10 \% \mathrm{MVC}$ in the 8 to $10-\mathrm{Hz}$ bandwidth. (F) Average amount of power in the force spectrum as a function of feedback intermittency at $10 \% \mathrm{MVC}$ in the 10 to $12-\mathrm{Hz}$ bandwidth.

creased as more visual information became available. Congruent with the power analysis, examination of the spectral slope showed that the force spectrum became broader as a function of visual information. Overall, these results are consistent with the postulation that visual motor processing is composed of stochastic and deterministic processes, which operate at frequency bands up to $12 \mathrm{~Hz}$, depending on the force level.

\section{DISCUSSION}

The experimental findings provide evidence that the visual motor processing of intermittent information is dependent on the isometric force level. The visual control of force output is organized by the multiple time scales of influence from 0 to $12 \mathrm{~Hz}$, rather than being due only to a low-frequency modal activity, as has previously been pro- 
Table 1

Linear Regression Coefficients for Amount of Power in 2-Hz Bins as a Function of Visual Intermittency

\begin{tabular}{lcccc}
\hline Bin & $\begin{array}{c}\text { Force Level } \\
\text { (\% MVC) }\end{array}$ & Intercept & Slope & $R^{2}$ \\
\hline $2 \mathrm{~Hz}$ & 5 & -0.319 & $-0.355^{*}$ & .900 \\
& 10 & -0.064 & $-0.408^{*}$ & .825 \\
& 25 & 0.562 & $-0.399^{*}$ & .896 \\
$4 \mathrm{~Hz}$ & 50 & 1.34 & $-0.319^{*}$ & .860 \\
& 5 & -1.53 & $-0.167^{*}$ & .809 \\
& 10 & -1.28 & $-0.208^{*}$ & .940 \\
& 25 & -0.589 & $-0.210^{*}$ & .907 \\
$6 \mathrm{~Hz}$ & 50 & 0.227 & $-0.107^{*}$ & .622 \\
& 5 & -2.17 & $-0.167^{*}$ & .809 \\
& 10 & -1.95 & $-0.087^{*}$ & .554 \\
$8 \mathrm{~Hz}$ & 25 & -1.18 & $-0.091^{*}$ & .659 \\
& 50 & -0.354 & $0.045^{*}$ & .583 \\
& 5 & -2.17 & $-0.072^{*}$ & .500 \\
& 10 & -2.33 & $-0.087^{*}$ & .554 \\
$10 \mathrm{~Hz}$ & 25 & -1.56 & $-0.081^{*}$ & .731 \\
& 50 & -0.711 & -0.028 & .115 \\
& 5 & -2.67 & -0.040 & .209 \\
& 10 & -2.48 & -0.031 & .144 \\
$12 \mathrm{~Hz}$ & 25 & -1.71 & $-0.095^{*}$ & .860 \\
& 50 & -0.857 & 0.032 & .317 \\
& 5 & -2.95 & 0.005 & .008 \\
& 10 & -2.68 & $-0.069^{*}$ & .810 \\
& 25 & -1.94 & $-0.086^{*}$ & .710 \\
& 50 & -0.974 & 0.010 & .040 \\
\hline
\end{tabular}

Note-MVC, maximal voluntary contraction. *Slope is significantly different from $0(p<.05)$.

posed (Freund \& Hefter, 1993; Miall et al., 1993; Slifkin et al., 2000). The findings showed that force variability decreased as a function of reduced intermittency of visual information and that enhancement in performance was related to an increase in irregularity of the force signal and a broadening of the force spectrum, both features of the structure of variability that are signatures of multiple time scales of control (Mayer-Kress et al., 2003; Mayer-Kress \& Newell, 2002).

\section{Intermittent Visual Information and the Amount of Force Variability}

The higher intermittency rates of visual information, or expressed another way, the reduced time intervals between the presentation of the visual information, led to a reduction in the amount of force variability (Slifkin et al., 2000). Enhancing the presentation rate of visual information facilitated performance, but it did so at a negatively accelerating rate. It appears that there was no additional enhancement of performance between the 12- and the 25.6-Hz intermittent vision conditions, a finding that is consistent with the long-held position that there is a limit on the rate at which visual information can be processed (cf. Carlton, 1992).

As was expected, the variability of force output was dependent on force level (Slifkin \& Newell, 1999, 2000). The standard deviation of force was smaller at lower force levels, and there was a systematic increase in variability between the $5 \%$ and the $50 \%$ MVC conditions. However, the results also showed that the signal-to-noise ratio (i.e., information transfer $[M / S D]$ ) had an inverted U-like trend within the 5\%-50\% MVC conditions, in that the highest signal-to-noise ratio was in the $25 \% \mathrm{MVC}$ condition. This is consistent with the findings from Slifkin and Newell $(1999,2000)$ that showed that the peak signal-to-noise ratio under a high-frequency presentation of visual information condition was at about $30 \%$ of MVC. This relation held in the present experiment over all levels of intermittent information presentation, except when the intermittency of visual information was at the lowest frequency rates $(0.2,0.4$ and $0.8 \mathrm{~Hz})$ of presentation. Thus, the processing of intermittent visual information interacts with the magnitude of force production in determining the amount of force variability.

\section{Visual Information and the Structure of Force Variability}

A key focus of our study was an examination of the structure of force variability (Newell \& Slifkin, 1998) as reflected in both time and frequency domain analyses of the force output as a function of intermittency of visual information. These analyses were consistent in revealing how the intermittency of visual information interacts with force level in determining the structure of force output. The findings across the structure of variability analyses showed that the presentation of less intermittent visual information (smaller time intervals of presentation) enhanced the complexity of the force signal. The force signal was more irregular in the time domain, with a smaller DFA scaling index and a larger spectral slope as a function of less intermittent information. All three of the functions of the structure of force variability showed a negatively accelerating rate of change with enhanced rate of visual information presentation. Again, these effects were present only at visual intermittency rates greater than $0.8 \mathrm{~Hz}$, and they interacted with force level. Thus, there was an interaction of intermittency of visual information and force level on the complexity of force output structure.

Comparison of the trends in the structure of force variability with those of the amount of variability $(S D)$ shows that there is not a one-to-one correspondence between these variables (Slifkin \& Newell, 1999). The structure variables (ApEn, DFA, and spectral slope) showed a continuous effect of force level, whereas the signal-to-noise ratio, as was discussed above, showed a discontinuity of influence around the $25 \% \mathrm{MVC}$ force level. This pattern of findings across the variables shows that the relation between the magnitude and the structure of force variability is not simply an inverse function and that the pattern of distributional measures may not parallel those determined from time-dependent analyses. Also and importantly, they provide additional evidence for the central hypothesis of this experiment, that there is an interaction between intermittency of visual information and force level in the control of force output. 


\section{Multiple Time Scales of Visual Information Processing}

The study provides strong support for the postulation that there are multiple time scales of processing in the visual control of force output and that these multiple time scales lead to the interpretation of continuous control of force output (Mayer-Kress \& Newell, 2002). The central finding of the experiment was a triple interaction in the average power of the frequency spectrum as a function of information intermittency, force level, and bandwidth of force output. Specifically, the effect of intermittency was less at the higher frequency bands, and this effect also interacted with force level. There was a nonmonotonic relationship between force level and the effect of visual intermittency, since the amount of power in the higher frequency bands was influenced by intermittency only in the $10 \%$ and $25 \%$ MVC conditions.

Although most of the change in the power of the force signal due to intermittency of visual information is in the 0 to $2-\mathrm{Hz}$ bandwidth (Freund \& Hefter, 1993; Miall et al., 1993; Slifkin et al., 2000), there are significant effects of intermittency of visual feedback out to at least $12 \mathrm{~Hz}$, although importantly, these effects are found only in the $10 \%$ and $25 \%$ MVC conditions. Multiple time scales of influence in force production are enhanced under the less intermittent vision conditions and the higher levels of force production. There is not a modal frequency that drives the force output but, rather, multiple time scales of influence, at least up to about $12 \mathrm{~Hz}$, that are influenced by intermittency of visual information and force level. The multiple time scales in the force signal also arise from the more rapid cutaneous and proprioceptive inputs, and it appears that visual information integrates with information from these sensory systems up to at least $12 \mathrm{~Hz}$.

It appears that presentation of less intermittent visual information leads to the utilization of higher frequency feedback and feedforward processes of force output (Desmurget \& Grafton, 2000). These processes cannot be separated with the present experimental design and analyses, and in principle, they may not be easily separated in any protocol. Nevertheless, the present study shows that the level of intermittent visual information interacts with force level to modulate the strength of the long-range correlations evident in the frequency structure of the force output.

In summary, the intermittency of visual information interacts with force level in organizing the visual motor processing of isometric control. And there are multiple time scales of force output that are mediated by the interaction of force level and the intermittency of visual information. The multiple time scales of force output lead to the interpretation of continuous control in force production (Newell, Broderick, Deutsch, \& Slifkin, 2003), although the stochastic nature of this mode of control is strongly influenced by the intermittency of visual information and force level. In other words, the changing probabilistic frequency structure of the force output gen- erates a smooth trajectory of force over time and invites the observation that visual control is continuous. This observation is consistent with the position of Mulder and van Galen (1995) that the question of continuous versus discrete processing maybe a false dichotomy, at least in an operational perspective.

\section{REFERENCES}

Adams, J. A., \& Dijkstra, S. (1966). Short-term memory for motor responses. Journal of Experimental Psychology, 71, 314-318.

Broadbent, D. E. (1958). Perception and communication. London: Pergamon.

Carlton, L. G. (1981). Processing visual feedback information for movement control. Journal of Experimental Psychology: Human Perception \& Performance, 7, 1019-1030.

CARLTON, L. G. (1992). Visual processing time and the control of movement. In L. Proteau \& D. Elliott (Eds.), Vision and motor control (pp. 3-31). Amsterdam: Elsevier.

Desmurget, M., \& Grafton, S. (2000). Forward modeling allows feedback control for fast reaching movements. Trends in Cognitive Sciences, 4, 423-431.

DeUtsch, K. M., \& Newell, K. M. (2001). Age differences in noise and variability of isometric force production. Journal of Experimental Child Psychology, 80, 392-408.

ELLIOTT, D. (1990). Intermittent visual pickup and goal directed movement: A review. Human Movement Science, 9, 531-548.

ElLiotT, D. (1992). Intermittent versus continuous control of manual aiming movements. In L. Proteau \& D. Elliott (Eds.), Vision and motor control (pp. 33-48). Amsterdam: Elsevier.

Elliott, D., Carson, R. G., Goodman, D., \& Chua, R. (1991). Discrete versus continuous visual control of manual aiming. Human Movement Science, 10, 393-418.

FrEUND, H.-J., \& HEFTER, H. (1993). The role of the basal ganglia in rhythmic movement. In T. N. H. Narabayashi, N. Yanagisawa, \& Y. Mizuno (Eds.), Advances in neurology (Vol. 60, pp. 88-92). New York: Raven.

Gilden, D. L., Thorton, T., \& Mallon, M. W. (1995). $1 / f$ noise in human cognition. Science, 267, 1837-1839.

GrEen, S. B., \& SALKIND, N. J. (2003). Using SPSS for Windows and Macintosh (3rd ed). Upper Saddle River, NJ: Prentice-Hall.

Hausdorff, J. M., Mitchell, S. L., Firtion, R., Peng, C. K., CudKowICZ, M. E., \& WEI, J. Y. (1997). Altered fractal dynamics of gait: Reduced stride-interval correlations with aging and Huntington's disease. Journal of Applied Physiology, 82, 262-269.

Keele, S. W., \& Posner, M. I. (1968). Processing of visual feedback in rapid movements. Journal of Experimental Psychology, 70, 387-403.

Khan, M. A., Lawrence, G., Fourkas, A., Franks, I. A., Elliott, D., \& Pembroke, S. (2003). Online versus offline processing of visual feedback in the control of movement amplitude. Acta Psychologica, 113, 83-97.

LIPSITZ, L. A. (1995). Age-related changes in the "complexity" of cardiovascular dynamics: A potential marker of vulnerability to disease. Chaos, 5, 102-109.

THE MATHWORKS, INC. (1999). Matlab (Version 6.1). Natick, MA: Author. Mayer-Kress, G., Deutsch, K. M., \& Newell, K. M. (2003). Modeling the control of isometric force production with piece-wise linear, stochastic maps of multiple time-scales. Fluctuation \& Noise Letters, 3, L23-L29.

MaYer-Kress, G., \& Newell, K. M. (2002). Stochastic iterative maps with multiple time-scales for modeling human motor behavior. Nonlinear Phenomena in Complex Systems, 4, 1-8.

Miall, R. C., WeIR, D. J., \& Stein, J. F. (1993). Intermittency in human manual tracking. Journal of Motor Behavior, 25, 53-63.

MiLleR, J. (1988). Discrete and continuous models of human information processing: Theoretical distinctions and empirical results. Acta Psychologica, 67, 191-257.

MULDER, G., \& VAN GALEN, G. P. (1995). How continuous are discrete notions of information processing? Acta Psychologica, 90, 1-8. 
Newell, K. M., Broderick, M. P., Deutsch, K. M., \& Slifkin, A. B. (2003). Task goals and change in dynamical degrees of freedom with motor learning. Journal of Experimental Psychology: Human Perception \& Performance, 29, 379-387.

NewEll, K. M., \& SLIFKIN, A. B. (1998). The nature of movement variability. In J. P. Piek (Ed.), Motor behavior and human skill: A multidisciplinary approach (pp. 143-160). Champaign, IL: Human Kinetics.

Newell, K. M., Slobounov, S. M., Slobounova, E. S., \& MoleNAAR, P. C. M. (1997). Stochastic processes in postural center-ofpressure. Experimental Brain Research, 113, 158-164.

Peng, C.-K., Buldyrev, S. V., GoldberGer, A. L., Havlin, S., SiMONS, M., \& STANLEy, H. E. (1993). Finite size effects on long-range correlations: Implications for analyzing DNA sequences. Physics Review E, 47, 3730-3733.

PINCUS, S. M. (1991). Approximate entropy as a measure of system complexity. Proceedings of the National Academy of Sciences, $\mathbf{8 8}$, 2297-2301.

SANDERS, A. F. (1990). Issues and trends in the debate on discrete vs. continuous processing of information. Acta Psychologica, 74, 123-167.

Slifkin, A. B., \& Newell, K. M. (1999). Noise, information transmission and force variability. Journal of Experimental Psychology: Human Perception \& Performance, 25, 837-851.

SLIFKIN, A. B., \& NewELl, K. M. (2000). Variability and noise in continuous force production. Journal of Motor Behavior, 32, 141-150.

Slifkin, A. B., VaillancourT, D. E., \& Newell, K. M. (2000). Intermittency in the control of continuous force production. Journal of Neurophysiology, 84, 1708-1718.
Smith, W. M., \& Bowen, K. (1980). The effects of delayed and displaced visual feedback on motor control. Journal of Motor Behavior, 12, 91-101.

THOMSON, J. A. (1980). How do we use visual information to control locomotion? Trends in Neurosciences, 3, 247-250.

THOMson, J. A. (1983). Is continuous visual monitoring necessary in visually guided motion? Journal of Experimental Psychology: Human Perception \& Performance, 9, 427-443.

Vaillancourt, D. E., Larsson, L., \& Newell, K. M. (2003). Effects of aging on force variability, single motor unit discharge patterns, and the structure of 10,20, and $40 \mathrm{~Hz}$ EMG activity. Neurobiology of Aging, 24, 25-35.

VINCE, M. A. (1948). Corrective movements in a pursuit task. Quarterly Journal of Experimental Psychology, 1, 85-103.

von Hofsten, C., \& LeE, D. (1982). Dialogue on perception and action. Human Movement Science, 1, 125-138.

WARD, L. M. (2002). Dynamical cognitive science. Cambridge, MA: MIT Press.

WoODWORTH, R. S. (1899). The accuracy of voluntary movement. Psychological Review, 3, 1-119.

ZelazniK, H. N., HaWKins, B., \& Kisselburgh, L. (1983). Rapid visual feedback processing in single-aiming movements. Journal of Motor Behavior, 15, 217-236.

(Manuscript received October 23, 2003; revision accepted for publication June 7,2004 .) 